\title{
A microprocessor- (Heath ET-3400) based backup control system for laboratory experiments
}

\author{
W. LLOYD MILLIGAN and ANTHONY RICHARDSON \\ Neuroscience Laboratory, Veteran's Administration Hospital, Columbia, South Carolina 29201
}

\begin{abstract}
This paper describes a psychology laboratory experimental control system that utilizes a PDP-11/10 minicomputer to perform both instrument control and data acquisition functions. The minicomputer is backed up by a Heath ET-3400 microprocessor trainer system which performs control functions only. Major components of the hardware and software comprising this system are described.
\end{abstract}

Psychophysiological experiments in the Neuroscience Laboratory, Veteran's Administration Hospital, Columbia, South Carolina, are automated by a Digital Equipment Corporation PDP-11/10 minicomputer with an LPS-11 lab peripheral system. The computer performs both control and data acquisition functions, using the LPS-11 digital input/output ( $/ / 0)$ registers, eightchannel multiplexor, and analog/digital (A/D) converter. Data sensed from laboratory instruments are stored in memory or on scratch disk files until the end of a test session (typically about $90 \mathrm{~min}$ ), when they are analyzed by a background program. Upon completing the analysis, the program writes session parameters (classification variable values and dependent variable means) to permanent data files.

Development of this system has evolved continuously since acquisition of the PDP-11 in November 1975. At first, the minicomputer was used for control functions only, and these were shared by external hardwired logic circuits (BRS Digibits). The external logic also served as a backup system whenever the PDP-11 failed. With implementation of computer control, and later automatic data acquisition, the complexity of experiments increased. The relative ease with which parameters could be varied in software enticed us to make modifications that were not readily duplicated in the hardwired backup system. Moreover, with increasing disuse and hybridization, the reliability of the backup system suffered. It eventually became apparent that a more dependable backup system was needed. Renovation of procedure control rooms in the spring of 1978 provided a favorable opportunity to replace this system with a microprocessor-based system.

Two important considerations influenced the overall design of the backup control system. First, it was evident that the system should be microprocessor based, in order to preserve the flexibility of varying experimental parameters in software. A second consideration was the requirement that both systems be capable of controlling the same external laboratory (stimulus and response) instruments, that is, those used in experimental procedures. The problem of controlling identical lab instruments by two different computer systems was solved by designing "front ends" for each instrument. Thus, we designed a polygraph controller, shock stimulator controller, tone generator/amplifier controller, and set switch (Milligan, 1977) controller. The front ends each presented a single standard TTL load per signal line to the controlling device. One other device was needed to distribute the signal lines. This card, the distribution module, performs minimal logic functions in conveying computer or microprocessor outputs to the instrument controllers.

The choice of microprocessor was influenced by a third consideration. It was felt that the Heathkit ET3400 (Motorola M6800) trainer would serve the dual purpose of teaching the principles of microprocessor control systems and providing the central component of the backup system. In retrospect, we are satisfied with this choice, as many examples and exercises in the Heath training course are directly applicable to the psychology laboratory situation. Subsequent experience with the Commodore PET 2001 microcomputer suggests that it also would serve well as a psychology laboratory control computer. The PET has an IEEE bus interface and a general-purpose user port which consists of the B side of a 6520 peripheral interface adapter (PIA). The PET would be a more suitable choice for the user who wishes to program his application in an advanced language with a minimum of hardware and software development costs.

Figure 1 shows the arrangement of components in our laboratory. All components to the right of, and including, the distribution module are common to both the PDP-11 and ET-3400 control systems, with the exception of the dashed line at the top. This line represents eight channels of analog data from the polygraph to the PDP-11.

Inputs to the distribution module are conveyed via a 25-conductor ribbon cable that plugs directly into the 


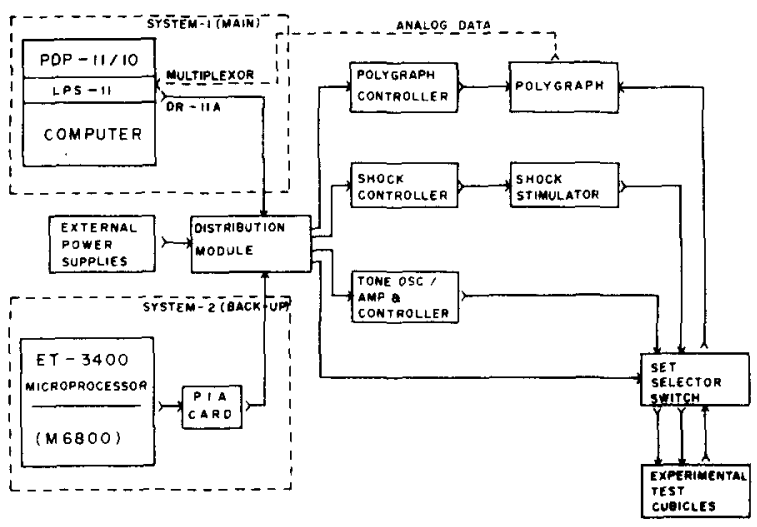

Figure 1. Arrangement of components in Neuroscience Laboratory, Veteran's Administration Hospital, Columbia, South Carolina.

LPS-11 DR-A 16-bit output register or into an identical 25-pin socket (Cinch DB25S) wired to the 6821 peripheral interface adapter (PIA) outputs. We substituted the newer and less expensive 6821 for the 6820 PIA furnished with the Heath training course. The 6821 is a programmable device, and in the present application all $16 \mathrm{I} / \mathrm{O}$ lines are initialized for output. Software for the ET-3400 is designed so that there is a $1: 1$ correspondence of definition between individual 6821 output lines (i.e., bits) and the same numbered lines of

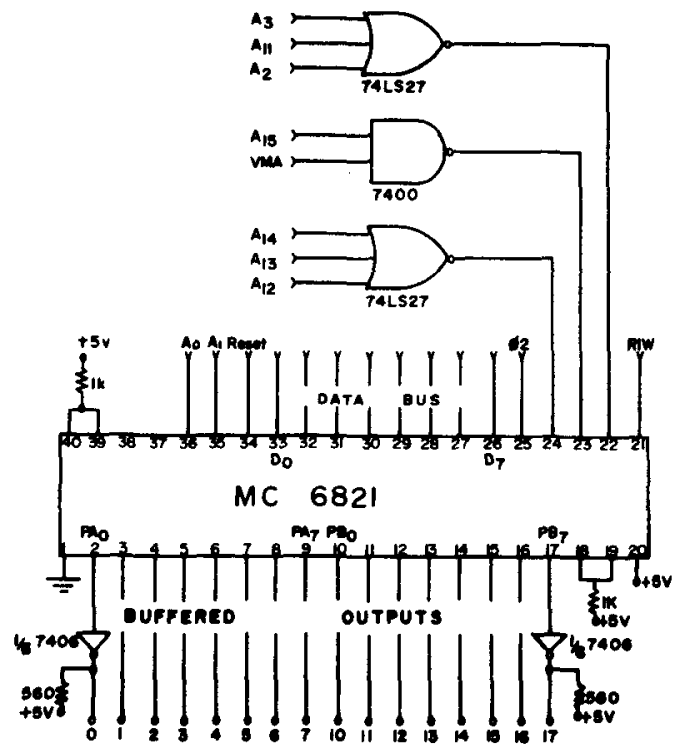

Figure 2. the DR-A. For example, Bit 0 of the low-order byte represents the "trial interval," Bit 1 is CS on, Bit 2 is test-trial select, and so forth. All definitions are "low enable"; the final output stage of the DR-A is an opencollector buffer (7416) normally pulled high. Interval timing is accomplished identically by both systems. In the PDP-11, the system time-of-day $(60-\mathrm{Hz})$ clock is invoked by a .TWAIT macro; in the ET-3400, a $60-\mathrm{Hz}$ interrupt clock is included in the control program. The sequence of time intervals is embedded in a set of nested counters: SETS $\subset$ 8-TRIAL BLOCK $\subset$ 8-BLOCK SESSION. A minor disadvantage of the microprocessor system is that the control program and experimental constants (time intervals, counters, etc.) must be keyed in manually. Since it is primarily an emergency backup system, this drawback can be tolerated.

Figure 2 shows the interface circuitry that connects the ET-3400 system to the distribution module. All of the bus connections (address, data, and control lines) on the ET-3400 are fully buffered.

The distribution module and each controller is constructed on a separate prototyping PC board, and all boards are contained within a single shielded enclosure (BUD AC1428). Control signals are conveyed from the distribution module to the controller cards via doubleended DIP jumpers that plug into 14-pin sockets on each board. Outputs to the instruments leave the cards at the foil-edge connectors.

A typical control program for executing one of the experiments performs all the control functions of a two-tone autonomic conditioning experiment for four animals simultaneously. The analogous control and data acquisition program for the PDP-11 is approximately $4 \mathrm{~K}$ bytes long. In the ET-3400 backup system the control part alone is approximately 300 bytes long.

In summary, the microprocessor-based system we have described is an economical and reliable backup for the control functions of the laboratory minicomputer. In addition, a level of flexibility is attained that would be difficult to duplicate with hardwired logic. The system, as described, retains the possibility of future modification and expansion to accommodate experiments of increasing complexity.

\section{REFERENCE}

Milligan, W. L. Electromechanical switching of preamplifier input leads: A method of increasing the usefulness of electrophysiological recording instruments. Psychophysiology, 1977, 14, 507.508. 